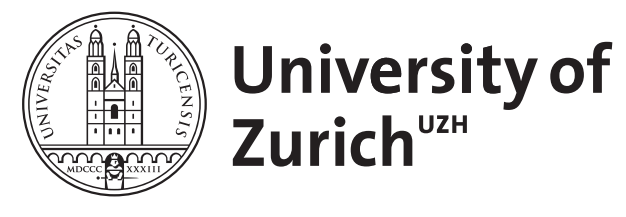

\title{
Weibliche Sexualität und sexuelle Störungen
}

\author{
Leeners, Brigitte
}

\begin{abstract}
Die weibliche Sexualität wird durch ein komplexes Netzwerk psycho-sexueller und körperlicher Faktoren beeinflusst. Sexuelle Störungen sind häufig und die meisten dieser Störungen haben bei einer adäquaten Unterstützung und ausreichenden Motivation der Betroffenen eine sehr gute Prognose. Naturgemäß beeinflussen gynäkologisch-geburtshilfliche Veränderungen und Krankheitsbilder die Sexualität, sodass Patientinnen sorgfältig über potenzielle Wechselwirkungen informiert werden sollten. Die gynäkologisch-geburtshilfliche Fachkompetenz beinhaltet zahlreiche Komponenten, welche Frauen/Paaren helfen können, eine erfüllende Sexualität zu gestalten. Lässt sich hierüber eine unzureichende Verbesserung erzielen, so bieten verschiedene sexualtherapeutische Konzepte wertvolle Optionen.
\end{abstract}

DOI: https://doi.org/10.1007/s00129-016-4007-x

Posted at the Zurich Open Repository and Archive, University of Zurich

ZORA URL: https://doi.org/10.5167/uzh-146941

Journal Article

Published Version

Originally published at:

Leeners, Brigitte (2017). Weibliche Sexualität und sexuelle Störungen. Gynäkologie, 1(50):55-65.

DOI: https://doi.org/10.1007/s00129-016-4007-x 


\section{Redaktion}

T. Dimpfl, Kassel

W. Janni, Ulm

R. Kreienberg, Landshut

N. Maass, Kiel

O. Ortmann, Regensburg

B. Sonntag, Hamburg

K. Vetter, Berlin

R. Zimmermann, Zürich

CrossMark

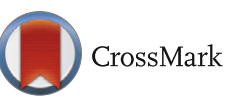

\section{Online teilnehmen}

\section{Punkte sammeln auf}

\section{CME.SpringerMedizin.de}

\section{Teilnahmemöglichkeiten}

Die Teilnahme an diesem zertifizierten Kurs ist für 12 Monate auf CME.SpringerMedizin.de möglich. Den genauen Teilnahmeschluss erfahren Sie dort.

Teilnehmen können Sie:

- als Abonnent dieser Fachzeitschrift, - als e.Med-Abonnent.

\section{Zertifizierung}

Diese Fortbildungseinheit ist zertifiziert von der Ärztekammer Nordrhein gemäß Kategorie $D$ und damit auch für andere Ärztekammern anerkennungsfähig. Es werden 3 Punkte vergeben.

\section{Anerkennung in Österreich}

Gemäß Diplom-FortbildungsProgramm (DFP) werden die auf CME.SpringerMedizin.de erworbenen Fortbildungspunkte von der Österreichischen Ärztekammer 1:1 als fachspezifische Fortbildung angerechnet (\$26(3) DFP Richtlinie).

\section{Kontakt}

Springer Medizin Kundenservice

Tel. 08007780777

E-Mail:kundenservice@springermedizin.de

\section{Empfohlen von derDAGG

\section{CME Zertifizierte Fortbildung}

Brigitte Leeners

Clinic for Reproductive Endocrinology, Zurich, Schweiz

\section{Weibliche Sexualität und sexuelle Störungen}

\section{Zusammenfassung}

Die weibliche Sexualität wird durch ein komplexes Netzwerk psycho-sexueller und körperlicher Faktoren beeinflusst. Sexuelle Störungen sind häufig und die meisten dieser Störungen haben bei einer adäquaten Unterstützung und ausreichenden Motivation der Betroffenen eine sehr gute Prognose. Naturgemäß beeinflussen gynäkologisch-geburtshilfliche Veränderungen und Krankheitsbilder die Sexualität, sodass Patientinnen sorgfältig über potenzielle Wechselwirkungen informiert werden sollten. Die gynäkologisch-geburtshilfliche Fachkompetenz beinhaltet zahlreiche Komponenten, welche Frauen/Paaren helfen können, eine erfüllende Sexualität zu gestalten. Lässt sich hierüber eine unzureichende Verbesserung erzielen, so bieten verschiedene sexualtherapeutische Konzepte wertvolle Optionen.

\section{Schlüsselwörter}

Hormone $\cdot$ Beratung $\cdot$ Sexuelle Aktivität $\cdot$ Lebensqualität $\cdot$ Schwangerschaft 


\section{Lernziele}

Nach der Lektüre dieses Beitrags ...

- kennen Sie die Grundzüge der weiblichen Sexualphysiologie.

- haben Sie einen Überblick über die wichtigsten sexuellen Störungen der Frau.

- können Sie Ihren Patientinnen verschiedene Hilfestellungen zur Gestaltung einer erfüllenden Sexualität geben.

- kennen Sie Indikationen und den Ablauf einer Sexualtherapie.

\section{Hintergrund}

Beim Menschen hat Sexualität neben der Fortpflanzung weitere Funktionen

Vermeintlich „normales” sexuelles Verhalten zu definieren kann Scham und Schuldgefühle begünstigen

Hyperämie ist essenziell für eine weitere Erregungssteigerung

Subjektiv erlebtes Erregungsniveau und physiologische Parameter korrelieren nur schwach
Der Mensch ist eines der wenigen Lebewesen, welches losgelöst von der Reproduktion z. B. während der Schwangerschaft oder postmenopausal, sexuell aktiv ist. Dies deutet darauf hin, dass Sexualität beim Menschen neben der Fortpflanzung weitere Funktionen hat, beispielsweise für die Stabilität einer Paarbeziehung [1, 2, 3] und die Gesundheit insgesamt $[4,5]$.

\section{Physiologische Grundlagen}

Individuell bestehen große Unterschiede in Bezug auf das Ansprechen verschiedener erogener Zonen, die Art/Intensität körperlicher Reaktionen etc. Der Versuch vermeintlich objektiv normales sexuelles Verhalten zu definieren, sollte im Umgang mit Patientinnen unterbleiben, da eine solche Definition Scham und Schuldgefühle begünstigen kann [6].

\section{Weibliche sexuelle Reaktion}

Ein von Masters und Johnson [7] entwickeltes Modell stellt die weibliche sexuelle Reaktion als lineares Modell mit insgesamt 4 Phasen dar:

\section{Erregungsphase}

Mit sexueller Erregung wird die Perfusion der Beckenorgane gesteigert und führt zu einem Anschwellen von Klitoris/Labien sowie zu einer Aufweitung und Befeuchtung (Lubrikation) der Vagina. Diese Hyperämie ist essenziell für eine weitere Erregungssteigerung. Sie bedingt außerdem eine leichte Vergrößerung der Brust/Mamillen und eventuell Hautrötungen („sex flush“). Parallel beschleunigen sich Puls und Atemfrequenz.

\section{Plateauphase}

Mit zunehmender Erregung kommt es zur Anspannung der Beckenbodenmuskulatur und zu einer Konzentration der Körperwahrnehmung auf die Geschlechtsorgane. Zwischen subjektiv erlebtem Erregungsniveau und physiologischen Messungen wie Perfusion, Lubrikation etc. besteht nur

\section{Female sexuality and sexual disorders}

\begin{abstract}
Female sexuality is regulated by a complex network of psychosexual and physical factors. Sexual disorders are common; however, with adequate professional support and motivation, most patients have a good prognosis. As by nature gynaecological and obstetrical changes as well as diseases influence sexuality, patients should be informed about any effect on sexuality and vice versa. Gynaecological and obstetrical knowledge may help women/couples to create a fulfilling sexuality. Different concepts for sexual therapy represent valuable options for improvement if counseling alone does not lead to satisfactory results.
\end{abstract}

\section{Keywords}

Hormones · Counseling · Sexual activity · Quality of life · Pregnancy 


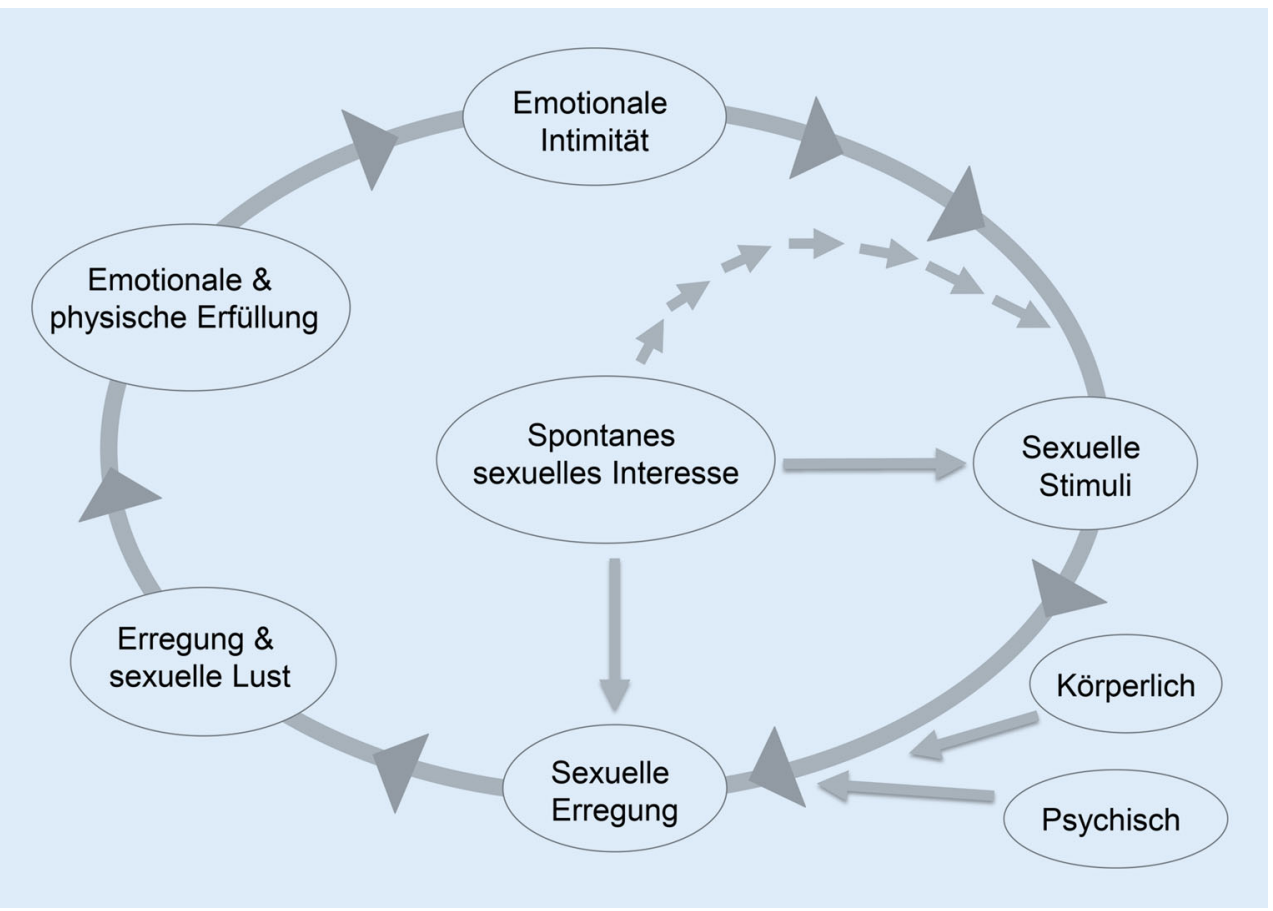

Abb. 1 \& Sexueller Zyklus nach Basson [13]

eine schwache Korrelation. Der Übergang von der Erregungs- zur Plateauphase ist fließend, eine Trennung beider Phasen ist umstritten.

\section{Orgasmus}

Während des Orgasmus kontrahieren Beckenboden und Uterus während etwa $15 \mathrm{~s}$ unwillkürlich und rhythmisch. Parallel wird bei einigen Frauen Sekret aus den paraurethralen Drüsen (SkenDrüsen) über die Harnröhre freigegeben, was z. T. fälschlicherweise als Urinabgang interpretiert wird. Der Orgasmus wird mental von Kontrollverlust und Gefühlen der Erfüllung begleitet. Eine Stimulation der Klitoris ist für viele Frauen die leichteste Möglichkeit, zum Orgasmus zu gelangen [8]. Während weit über $90 \%$ der Frauen durch Selbstbefriedigung einen Orgasmus erreichen, führt Geschlechtsverkehr lediglich bei etwa $30 \%$ aller Frauen zuverlässig zum Orgasmus [8, 9]. Dabei erschweren allerdings methodische Unterschiede - insbesondere die fehlende Exploration einer begleitenden direkten Klitorisstimulation beim Geschlechtsverkehr - den exakten Vergleich verschiedener Studien. Bei Frauen ist das Erreichen eines Orgasmus einer von mehreren gleichwertigen Parametern, welche die Qualität der sexuellen Beziehung bestimmen [10, 11].

Entgegen der vielfach zitierten, jedoch nie belegten „up-suck theory“, nach der Uteruskontraktionen beim Orgasmus die Aufnahme von Spermien in die Gebärmutter begünstigen sollen, erhöht ein Orgasmus der Frau die Wahrscheinlichkeit für eine Schwangerschaft nach heutigem Wissen nicht [12].

\section{Refraktärphase}

Puls-, Atemfrequenz und Hyperämie mit den dadurch bedingten Veränderungen kehren auf das Ausgangsniveau zurück.

Aufgrund der Frage, ob ein lineares Modell der weiblichen Sexualität gerecht wird, wurden zirkuläre Modelle vorgestellt. Nach diesen beeinflussen sich spontanes sexuelles Interesse, emotionale Intimität, sexuelle Stimuli/Erregung/Lust, emotionale und körperliche Erfüllung etc. gegenseitig [13]. Das von Basson entwickelte Modell ( $\bullet$ Abb. 1) betont die Bedeutung emotionaler Intimität und der Qualität der partnerschaftlichen Beziehung sowie die Komplexität der weiblichen Sexualität. Neben spontanem sexuellem Interesse ist auch ein Bedürfnis nach emotionaler und/oder körperlicher Nähe ein Grund, einen sexuellen Kontakt zu initiieren. Auf dieser Basis kann Erregung auf vielfältige Weise (z. B. Gespräche, erotische Literatur/Filmszenen, emotionale
Der Orgasmus wird mental von Kontrollverlust und Gefühlen der Erfüllung begleitet

Das Erreichen eines Orgasmus ist nur einer von mehreren gleichwertigen Parametern für die Qualität der sexuellen Beziehung

Neben spontanem Interesse ist auch das Bedürfnis nach Nähe ein Grund für das Initiieren eines sexuellen Kontakts 
Vor einer Hysterektomie sollte die Bedeutung des Uterus für das Sexualleben thematisiert und exploriert werden

An der Regulation von Elastizität und Belastbarkeit der Vaginalwand beteiligte Proteine werden östrogenabhängig gebildet

Eine Behandlung postmenopausaler Frauen mit Testosteron kann zu einer Libidosteigerung führen

Libidoverlust ist eine der Hauptursachen für das Sistieren einer oralen Kontrazeption oder einen Präparatewechsel
Nähe, direkte körperliche Stimulation etc.) entstehen bzw. gesteigert werden. Dabei spielt die zunehmende Erregung eine entscheidende Rolle bei der Motivation den Kontakt zu intensivieren. Jede einzelne Komponente des Zyklus unterliegt psychischen und physischen Störfaktoren.

Die Bedeutung der Gebärmutter bei sexueller Erfüllung ist individuell unterschiedlich und wird durch die Funktion als Repräsentanzorgan (Weiblichkeit, Fruchtbarkeit) sowie die individuell variable Beteiligung z. B. von auf die Zervix ausgeübtem Druck/Kontraktionen der Gebärmutter am sexuellen Erleben bestimmt. Wird diese individuelle Situation vor einer eventuellen Hysterektomie exploriert und in die Beratung einbezogen, zeigt sich postoperativ eine höhere Zufriedenheit in Bezug auf die Sexualität.

\section{Endokrinologie der Sexualität}

Östrogene nehmen eine entscheidende Rolle bei den körperlichen Voraussetzungen für die weibliche Sexualität ein $[14,15]$ : Sie regulieren die Vaskularisierung von Vaginalwand, Klitoris und Klitorisschwellkörpern. Eine adäquate Lubrikation- die Befeuchtung der Scheidenwand über Transsudation durch eine Erhöhung der epithelialen Permeabilität mittels Prostazyklinen - ist östrogenabhängig. Östrogene bestimmen die Dicke der Vaginalwand und führen durch ein aufgrund der Döderlein-Flora leicht saures Vaginalmilieu zu einem Schutz gegenüber Vaginalinfekten. Darüber hinaus werden an der Regulation von Elastizität und Belastbarkeit der Vaginalwand beteiligte Proteine östrogenabhängig gebildet. Die Dichte und Struktur glatter Muskelzellen sowie die Innervation der Vaginalwand werden maßgeblich durch Östrogen reguliert. Während einzelne Untersuchungen auf eine günstige Wirkung auf spezifische sexuelle Parameter deuten, zeigt die Mehrzahl der Untersuchungen keinen direkten Effekt.

Testosteron wird auch bei Frauen im Zusammenhang mit sexueller Libido diskutiert. Bei Frauen sinkt der Testosteronspiegel physiologisch ab dem 32. Lebensjahr ohne eine perimenopausale Änderung der Dynamik. Zwischen Serumspiegeln verschiedener Androgene und der weiblichen Libido besteht keine Korrelation [16]. Eine Behandlung postmenopausaler Frauen mit Testosteron kann zu einer Libidosteigerung führen. So stellt eine an die physiologischen niedrigen Testosteronspiel angepasste Therapie bei Libidostörungen eine Behandlungsoption dar [17]. Eine mögliche Indikation für eine Testosterontherapie ist ein ausgeprägter Libidoverlust nach bilateraler Ovarektomie bei jungen prämenopausalen Frauen. Im deutschsprachigen Raum ist derzeit kein Testosteronmonopräparat für diese Indikation zugelassen. Insgesamt sollte wegen des Nebenwirkungsprofils und unzureichender Langzeitdaten, z. B. in Bezug auf das Thromboserisiko oder ein Mammakarzinom, die Indikation für eine Testosterontherapie sehr sorgfältig geprüft werden.

Bei den Gestagenen kann die antiandrogene Partialwirkung eines oralen Kontrazeptivums bei diesbezüglich empfindsamen Frauen eine ungünstige Wirkung auf die Libido zeigen. Eine stimulierende Wirkung auf die Bildung von SHBG („sex hormone binding globulin“) kann unabhängig vom Gestagen zu einer Reduktion biologisch wirksamer Androgene führen. Obwohl bei vielen Frauen keine ungünstige Wirkung auf die Libido auftritt, ist ein Libidoverlust eine der Hauptursachen für das Sistieren einer oralen Kontrazeption oder den Wechsel auf ein anderes Präparat.

Nach einem Geschlechtsverkehr kommt es zu einem deutlichen Prolaktinanstieg, was mit reduzierter Lust auf einen erneuten Kontakt assoziiert ist [18]. Weitere Hinweise auf eine regulative Wirkung von Prolaktin bei der weiblichen Libido ergeben sich aus der klinischen Beobachtung, dass Frauen während der Stillphase, d. h. in einer Phase physiologisch deutlich höherer Prolaktinspiegel, oder bei Hyperprolaktinämie infolge eines Prolaktinoms oftmals über einen Libidoverlust klagen.

\section{Störungen der sexuellen Interaktion}

\section{Störungsbilder}

Weibliche Sexualität wird über ein komplexes Netzwerk psychosexueller und körperlicher Faktoren reguliert (• Abb. 2). Eine Übersicht zu den häufigsten sexuellen Störungen bei der Frau gibt - Tab. 1. Das Vorliegen einer Störung beinhaltet definitionsgemäß einen durch das Symptom bedingten Leidensdruck. Sexuelle Störungen sind in Auftreten und Ausprägungsgrad partner-, 


\section{Psychosoziale/ -sexuelle Voraussetzungen}

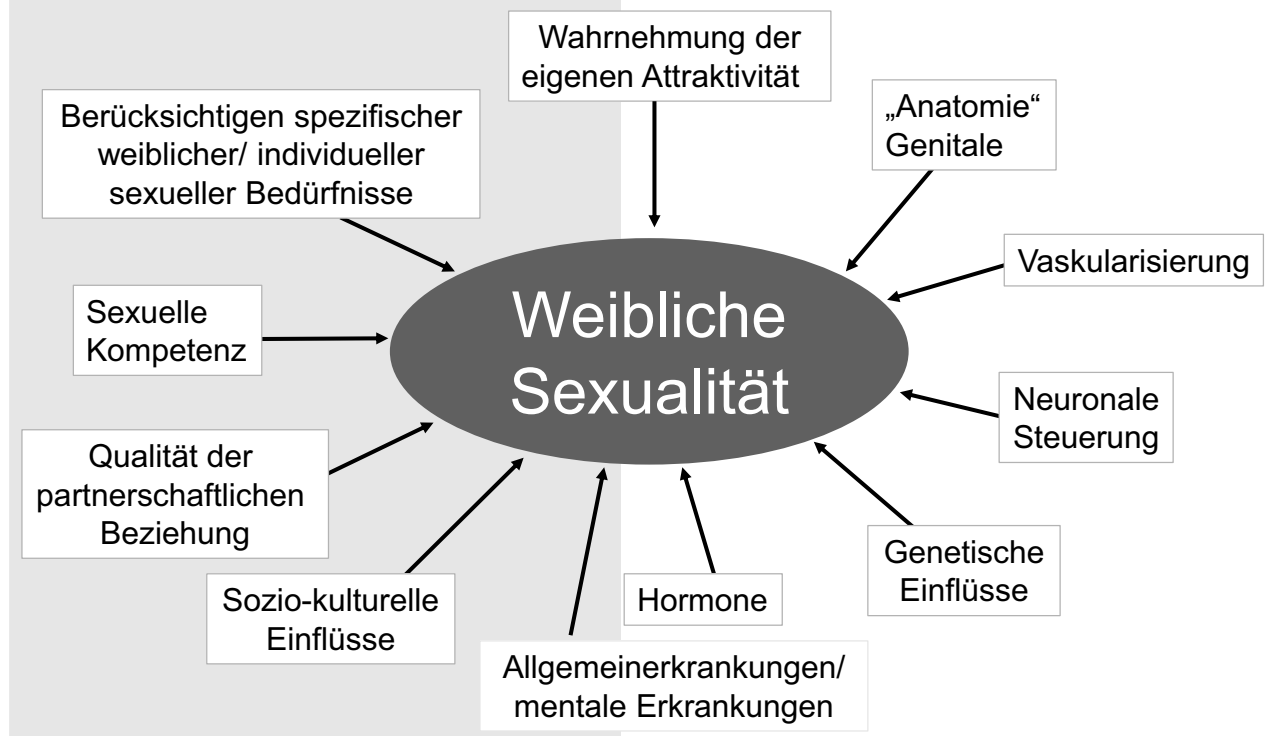

Abb. $2 \Delta$ Einflussfaktoren der weiblichen Sexualität: Der Mensch ist das einzige Wesen, welches Sexualität auch losgelöst von Reproduktion praktiziert. In der Schwangerschaft und der postmenopausalen Phase ist die Libido sehr komplex, da Wechselwirkungen zwischen einzelnen Symptomen (z. B. Erfüllung beeinflusst Lust, Grundstimmung beeinflusst Lust) bestehen. Folglich sind isolierte Parameter schwer zu erfassen.

Tab. 1 Häufigste Störungen der sexuellen

Interaktion

Sexuelle Lustlosigkeit (häufigste Störung)

Erregungsstörungen

Vaginismus

Dyspareunie (dritthäufigste Störung)

Orgasmusstörungen (zweithäufigste Störung)

\section{Körperliche Voraussetzungen}


werden. Unterschieden werden eine primäre, d.h. seit dem erstem Geschlechtsverkehr bestehende von einer sekundären Dyspareunie, z. B. nach sexuellen Traumatisierungen, Entbindungen, gynäkologischen Operationen etc. Die Differenzierung zwischen einer oberflächlichen („entry“), mittleren und tiefen Dyspareunie hilft bei der Evaluation pathophysiologischer Ursachen. Häufig treten Mischformen zwischen physiologisch, somatisch und psychosomatisch bedingten Schmerzen auf.

\section{Vaginismus}

Beim Vaginismus handelt es sich um eine unbewusste, individuell sehr unterschiedlich ausgeprägte Anspannung des Beckenbodens, die durch den Versuch Tampon, Finger bzw. Penis einzuführen, eine gynäkologische Untersuchung oder durch Gedanken an diese Situationen ausgelöst werden kann. Es handelt sich um eine rein psychogene Störung, welche über eine Verbesserung der Körperwahrnehmung im Genitalbereich im Rahmen einer Sexualtherapie eine exzellente Prognose hat.

\section{Orgasmusstörungen}

Häufig führen falsche Erwartungen/ Vorstellungen zu einer subjektiven Fehldiagnose

Auch psychiatrische Erkrankungen, v.a. Depressionen, sind häufige Mitursachen sexueller Störungen.

Sexuelle Störungen können eine Funktion für das psychische Gleichgewicht der Betroffenen haben

Stellungen sollten mit zunehmendem Schwangerschaftsverlauf angepasst werden
Kommt es trotz adäquater Stimulation zu keinem Orgasmus, so liegt eine Orgasmusstörung vor. Häufig führen falsche Erwartungen/Vorstellungen z. B. in Bezug auf das Ansprechen auf potenzielle sexuelle Reize jedoch zu einer subjektiven Fehldiagnose. Somit sollte zunächst abgeklärt werden, ob spezifische individuelle Bedürfnisse konkret benannt werden können und tatsächlich umgesetzt werden. Neben der sexuellen Kompetenz beeinflussen auch genetische Faktoren die Wahrscheinlichkeit für einen Orgasmus [22].

\section{Ursachen}

Störungen der sexuellen Interaktion sind häufig multifaktoriell bedingt. $\mathrm{Zu}$ den organischen Ursachen zählen Folgeerscheinungen chronischer Erkrankungen z. B. Diabetes mellitus, multiple Sklerose, Fehlbildungen des Genitales und körperliche Einschränkungen (Beweglichkeit/ Kondition). Auch psychiatrische Erkrankungen, insbesondere Depressionen, sind häufige Mitursachen sexueller Störungen. Iatrogene Ursachen umfassen allergische Reaktionen auf intravaginale Kontrazeptiva (Spermizide, Kondome), Nebenwirkungen von Medikamenten (z. B. Psychopharmaka, orale Kontrazeptiva, Antihistaminika, $\mathrm{H}_{2}$-Blocker, Diuretika, Antihypertensiva etc.) und Auswirkungen operativer, chemo- sowie radiotherapeutischer Behandlungen. Bei Verordnung systemischer hormoneller Kontrazeptiva sollte sorgfältig über eine ungünstige Wirkung auf die Libido aufgeklärt werden [23, 24, 25]

\section{Psychosoziale Ursachen}

Vielen Paaren fällt schwer, sich über sexuelle Bedürfnisse auszutauschen, was eine der Hauptursachen bei der Entstehung von Störungen darstellt. Sexuelle Störungen können eine Funktion bei der Stabilisierung des psychischen Gleichgewichts der Betroffenen haben. Hierunter fallen z. B. Folgen von Tabuisierung von Sexualität, Angst vor Kontrollverlust, Partnerverlust etc. Darüber hinaus ist eine Regulation der Paardynamik (Nähe-Distanz, Macht) eine potenzielle Funktion sexueller Störungen. Weitere Einflussfaktoren sind Erfahrungs- und Fertigkeitsdefizite, eine begrenzte Vertrautheit mit eigenen sexuellen Reaktionen und Erwartungs- bzw. Versagensängste. Eine auf weibliche Bedürfnisse wenig eingehende, auf den Koitus ausgerichtete Norm partnerschaftlicher Sexualität und Erfahrung sexualisierter Gewalt können sexuelle Störungen begünstigen [6].

\section{Sexualität und Schwangerschaft}

Tendenziell nehmen sexuelles Interesse und sexuelle Aktivität mit zunehmendem Gestationsalter ab, wobei interindividuelle Unterschiede stärker sind als intraindividuelle [26, 27]. In der komplikationslosen Schwangerschaft besteht (auch im letzten Trimenon) keine Kontraindikation gegen jede von beiden Partnern gewünschte Form von Sexualität, wobei Stellungen mit zunehmendem Schwangerschaftsverlauf angepasst werden sollten (keine Rückenlage, d. h. keine Missionarsstellung gegen Ende der Schwangerschaft). Grundsätzlich werden in Zusammenhang mit sexueller Aktivität ungünstige Auswirkungen auf das Abortrisiko, sexuell übertragbare Erkrankungen und 


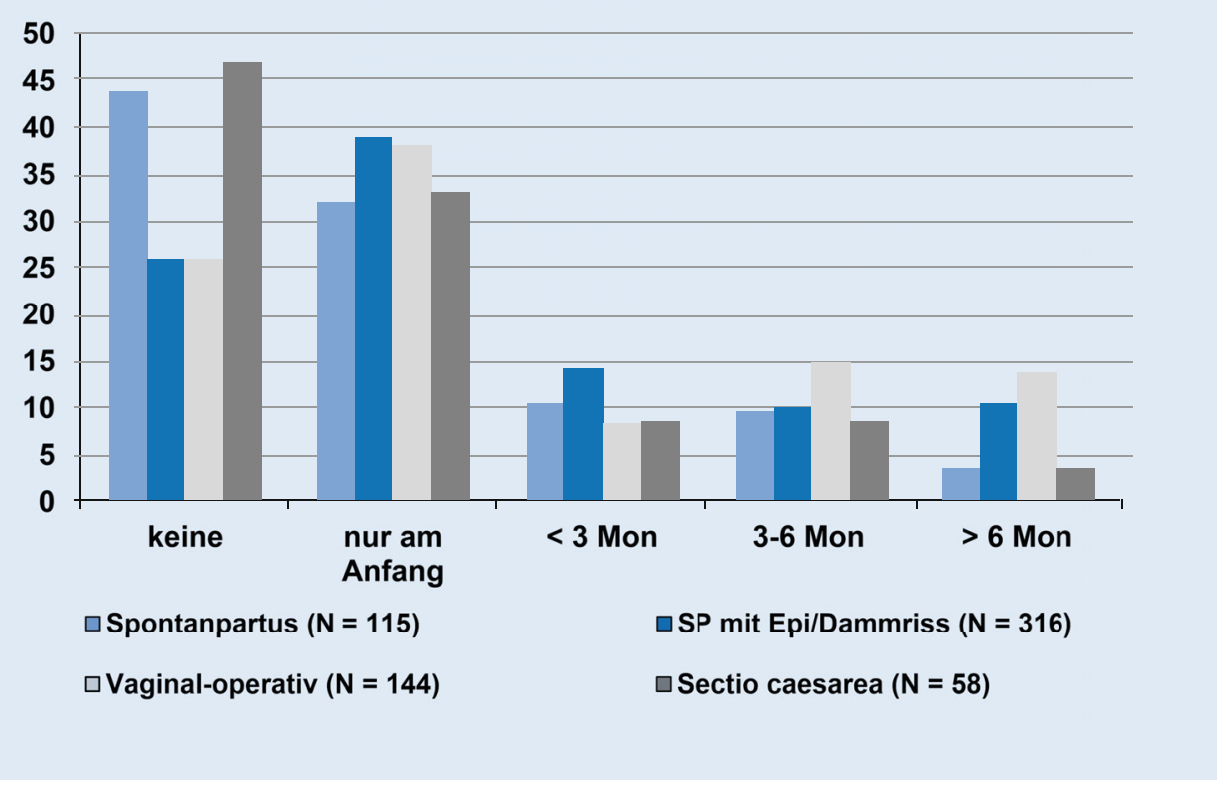

Abb. 3 A Postpartale Dyspareunie: Auswirkungen von Geburtsmodi. (Nach [29] und [30])

die Frühgeburtlichkeit befürchtet, wobei folgende pathophysiologischen Mechanismen diskutiert werden [28]:

- Reduktion der Plazentaperfusion durch Uteruskontraktionen

- Muttermundöffnung durch:

- mechanische Reizung von Vagina, Portio und Uterus

- Oxytocin $\uparrow$ bei Orgasmus und/oder Stimulation der Brustwarzen

- Freisetzung von Prostaglandinen aus den Eihäuten durch mechanische Reizung von Vagina, Portio und Uterus oder im Ejakulat (insbesondere $\mathrm{F}_{2} \alpha$ und $\mathrm{E}_{2}$ ). Die Wirkung dieser Prostaglandine ist abhängig vom Rezeptorstatus, der im Normalfall erst am Ende des dritten Trimenons eine ausreichende Dichte für einen geburtseinleitenden Effekt aufweist.

- Katecholamine $\uparrow$ bei emotionalem oder physischem Stress.

- Vorzeitiger Blasensprung durch:

- Destabilisierung der Eihaut über Kollagenase-ähnliche Enzyme im Ejakulat

- Amnioninfektionssyndrom aufgrund sexuell übertragbarer Infektionen.

Daten zum vorzeitigen Blasensprung sind widersprüchlich, tendenziell besteht keine signifikante Korrelation zwischen Geschlechtsverkehr/Orgasmus und vorzeitigem Blasensprung. Schwangeren mit einem vorzeitigen Blasensprung in der Anamnese ist jedoch von Geschlechtsverkehr abzuraten. Bei komplikationsloser Schwangerschaft besteht kein Zusammenhang zwischen Geschlechtsverkehr/Orgasmus und Frühgeburtlichkeit. Wie zu erwarten ist das Risiko bei vaginalen Infektionen erhöht.

Bei bis zu $90 \%$ der Mütter und Väter treten postpartal durch körperliche und/oder psychosoziale Veränderungen sexuelle Schwierigkeiten auf, welche bei einem Drittel längerfristig persistieren. Obwohl Frauen nach Spontanpartus mit Dammverletzung und Frauen nach vaginal-operativer Entbindung signifikant häufiger eine Dyspareunie als Frauen nach Spontanpartus ohne Dammverletzung oder nach Sectio caesarea angeben, zeigen sich 6 Monate postpartal keine signifikanten Unterschiede, d. h. eine Sectio caesarea ist keine geeignete Maßnahme zur Vermeidung einer postpartalen Dyspareunie (• Abb.3). Eine Thematisierung sexueller Aktivität sollte spätestens bei der Wochenbettabschlussuntersuchung erfolgen. Ursachen dieser Veränderungen sind

- auf somatischer Ebene

- eine Libido-reduzierende Wirkung von Prolaktin

- eine erhöhte Scheidentrockenheit unter Amenorrhö
Bei komplikationsloser Schwangerschaft stehen Geschlechtsverkehr/ Orgasmus und Frühgeburtlichkeit nicht in Zusammenhang

\section{Sexuelle Aktivität sollte spätestens} bei der Wochenbettabschlussuntersuchung thematisiert werden 
auf psychosozialer Ebene

- Schlafmangel

- wenig Zeit für eigene Interessen/Bedürfnisse

- ausgeprägter Körperkontakt zum Kind

- eventuelle Unzufriedenheit mit der körperlichen Attraktivität im Zusammenhang mit postpartalen körperlichen Veränderungen.

\section{Sexualität bei und nach Karzinomerkrankungen}

Eine Karzinomerkrankung hat psychische (emotionale Belastung durch Diagnose, Veränderungen der weiblichen/sexuellen Identität), soziale (Veränderung der partnerschaftlichen Beziehung, Alltagsgestaltung) und somatische Auswirkungen auf die Sexualität. Obwohl Sexualstörungen häufig erstmalig nach einer Karzinomtherapie auftreten, ist diese meist nicht die ausschließliche Ursache.

Sexuelles Interesse und sexuelle Aktivität sinken bei der Mehrzahl der Betroffenen zumindest kurzfristig. Während einer Chemotherapie besteht durch Nebenwirkungen meist geringes sexuelles Interesse. Je nach Erkrankung und Therapie begünstigen Veränderungen der Vaginalwand eine Dyspareunie. Chirurgische Interventionen an der Brust können neben Veränderungen der Perzeption der eigenen Attraktivität durch Sensibilitätsstörungen und Missempfindungen zur Reduktion von Brustzärtlichkeiten führen. Folgen eines Zervixkarzinoms werden durch die verbleibende Länge der Vagina und potenzielle Auswirkungen der Radiatio bestimmt. Hierzu zählen akut die radiogene Kolpitis und mittelfristig Kombinationseffekte der direkten Strahlenwirkung und einer eventuellen radiogen bedingten Menopause (Fibrosierung der Vaginalwand, 80-95 \% nach Afterloading, geringere Lubrikation, erhöhte Fragilität). Einer operativ oder radiogen bedingten Verkürzung/Obliteration der Vagina sollte primär mit Vaginaldilatoren entgegengewirkt werden. Sexuelle Aktivität ist - falls gewünscht und keine karzinombedingten Blutungen vorliegen - auch während einer Strahlentherapie möglich und kann hier einem Verkleben der Vaginalwand entgegenwirken. Patientinnen sollten darüber informiert werden, dass leichte Blutungen kein Zeichen eines Rezidivs sind oder ein Signal, den Geschlechtsverkehr aus medizinischen Gründen zu beenden. Auch 2-3 Jahre nach Erstbehandlung einer Karzinomerkrankung zeigen sich gehäuft sexuelle Störungen. Um nach Therapieabschluss eine Entwicklung zu einer erfüllenden Sexualität zu erleichtern, sollte frühzeitig über Auswirkungen auf die Sexualität informiert und auch Unterstützung im Intervall angeboten werden.

\section{Sexualberatung und Therapie}

\section{Beratung}

Offenheit gegenüber sexuellen Fragen und insbesondere ein aktives Gesprächsangebot durch den Frauenarzt/die Frauenärztin erleichtert es Patientinnen, sexuelle Schwierigkeiten anzusprechen. Konkrete direkte Fragen und Antworten werden von den meisten Patientinnen als erleichternd und schamreduzierend empfunden. Die Möglichkeit eines Gespräches über Sexualität kann in die systematische Anamnese integriert werden (z. B. „Sind Sie zufrieden mit Ihrer Sexualität?“, „Ist in Bezug auf Ihre Sexualität alles in Ordnung?") oder anhand eines Symptoms angesprochen werden, das sich bei der gynäkologischen Untersuchung gezeigt hat (z. B. „Mir ist aufgefallen, dass der Beckenboden bei der Untersuchung sehr angespannt und so der Scheideneingang recht eng war. Ist dies bei sexuellen Aktivitäten auch so? Wenn ja: Bereitet Ihnen dies Schwierigkeiten/ Stört Sie dies bei der Sexualität?“). Im Rahmen einer Sexualanamnese sollten Art, Häufigkeit und Entstehungsgeschichte (einschließlich körperlicher Grunderkrankungen, Medikamente) des sexuellen Problems unter Einbezug situations-, partner-, und praktikabhängiger Aspekte evaluiert werden $[31,32]$.

\section{Unterstützende Maßnahmen}

Eine Sexualberatung kann folgende Unterstützungsangebote umfassen:

- Informationen über den weiblichen Körper und die weibliche Sexualphysiologie 
- Unterstützung der Kommunikation über sexuelle Bedürfnisse

- Bei Medikamenten/Operation/chronischer Erkrankung sorgfältige Aufklärung über mögliche Auswirkungen auf die Sexualität

- Gleitmittel

- Wasserlösliche Gleitmittel sind besser geeignet als Gleitmittel auf Lipidbasis (leichter zu entfernen, geringeres Risiko für vaginale Infekte), jedoch dünnflüssiger und trocknen leichter ein

- Gleitmittel auf Silikonbasis sind besonders angenehm Cave: Die Lubrikation ist eine wichtige körperliche „Rückmeldung“ einer adäquaten Stimulation, daher unbedingt vermeiden, Gleitmittel an Stelle einer adäquaten Stimulation einzusetzen.

- Lokale und systemische Hormongaben entsprechend der klassischen Indikationen und Kontraindikationen.

- Eventuell Vermittlung einer Physiotherapie zum Erlernen einer bewussten Kontrolle d. h. Anund vor allem Entspannung des Beckenbodens (Reduktion einer Dyspareunie, Vaginismustherapie)

- Ggf. Umstellung einer medikamentösen Therapie auf Präparate mit geringeren Auswirkungen auf die Sexualität

\section{Spezielle Situationen}

\section{- Schwangerschaft}

- Ziel: möglichst erfüllende Partnerschaft (nicht ein möglichst aktives Sexualleben, auch Möglichkeit sexueller Abstinenz kann zufriedenstellende Lösung für beide Partner sein)

- Praktische Hinweise auf bestimmte sexuelle Aktivitäten/Stellungen/Unterstützung durch Kissen können hilfreich sein

- Bei Karzinomtherapie

- 80 \% der Patientinnen empfinden Aufklärung über Auswirkungen auf die Sexualität unzureichend $\rightarrow$ Auswirkungen auf Sexualität in prätherapeutische Aufklärung integrieren

- In der Akutphase werden Nebenwirkungen auf die Sexualität in der Regel gut toleriert, gewinnen mit zunehmendem zeitlichen Abstand zur Primäroperation jedoch an Bedeutung $\rightarrow$ Beratung zu Sexualität im Rahmen der Tumornachsorge anbieten

- Ärztinnen sollten auf Distanzierungstendenzen achten, denen Wissensdefizite zugrunde liegen (z. B. Ansteckungsphantasien bei Geschlechtsverkehr nach Zervixkarzinom)

\section{Sexualtherapeutische Konzepte}

Liegen spezifische Sexualstörungen (Libidoverlust, Vaginismus, Dyspareunie, etc.) vor und sind die Optionen einer Sexualberatung ohne eine zufriedenstellende Symptomverbesserung ausgeschöpft, so sollte eine Sexualtherapie durch eine/n ausgebildeten Sexualtherapeuten/in erfolgen. Die bei der Sexualberatung aufgeführten Elementen der Sexualanamnese werden dort vertieft und durch eine Exploration der psychosexuellen Entwicklung im Kontext der eigenen Biographie sowie der Bedeutung des sexuellen Problems für die eigene Psychodynamik bzw. die Paardynamik ergänzt. Die Sexualmedizin geht davon aus, dass beide Partner an der aktuellen Ausprägung des Symptoms eines Partners beteiligt sind. Für eine Sexualtherapie stehen verschiedene Konzepte zur Verfügung, die bei entsprechender Motivation innerhalb von Wochen/Monaten zu einer deutlichen Verbesserung führen können [6, 33]. Das Hamburger/Lübecker Modell („sensate focus") ist eine Weiterentwicklung des Konzeptes von Masters und Johnson [6]. Über verhaltenstherapeutische Paar- und Einzelübungen, die sich von Körperberührungen ohne erotische Elemente schrittweise bis zum Geschlechtsverkehr steigern, wird in einem sehr definierten Setting eine systematische Auflösung der den sexuellen Funktionsstörungen zugrunde liegenden Selbstverstärkungsmechanismen, eine bessere Körperwahrnehmung und eine Steigerung der sexuellen Kommunikationsfähigkeit angestrebt. Bei diesem Prozess werden an der sexuellen Störung beteiligte aktuelle und frühere psychosexuelle Faktoren (Ambivalenz zwischen Autonomie und Hingabe, Schutzfunktion des Symptoms etc.) integriert. Es handelt sich um ein primär paartherapeutisches Konzept, einzelne Sequenzen sind ebenfalls für ein Einzelsetting geeignet.

Die bei der Sexualberatung aufgeführten Elemente der Sexualanamnese werden in der Sexualtherapie vertieft

Das Hamburger/Lübecker Modell („,sensate focus") ist eine Weiterentwicklung des Konzeptes von Masters und Johnson 
Je nach Ausgangssituation bieten körpertherapeutische Elemente eine wichtige Ressource

Das Konzept ermöglicht eine präzise Diagnostik und fokussierte Arbeitshypothesen

In der systemischen Sexualtherapie können zirkuläre Fragen und paradoxe Interventionen eingesetzt werden

Die Übungen werden im privaten Setting durchgeführt und in den Therapiesitzungen besprochen. Je nach Ausgangssituation bieten körpertherapeutische Elemente eine wichtige Ressource zur Verbesserung der körperlichen Voraussetzungen einer erfüllenden Sexualität.

Eine Unterstützung nach Karzinomtherapie kann zusätzlich ein Kommunikationstraining (ggf. auch für die Aufnahme neuer Beziehungen), die Integration der veränderten Körperregion sowie eine Neugestaltung der sexuellen Aktivität entsprechend den veränderten sexuellen Bedürfnissen und Möglichkeiten beinhalten.

Das Sexocorporel ist ein auf der Basis von physiologischen, sexodynamischen, kognitiven und Beziehungskomponenten entwickeltes Konzept zur Evaluation der sexuellen Gesundheit und Behandlung sexueller Störungen. Die verschiedenen Komponenten ermöglichen eine präzise Diagnostik sowie fokussierte Arbeitshypothesen zur Verbesserung der Sexualität. Beispielsweise werden verschiedene Erregungsmodi (archaisch, mechanisch, archaisch-mechanisch, ondulierend und wellenförmig) definiert und therapeutisch genutzt. Das Modell nutzt Körperübungen als ein wesentliches therapeutisches Element.

Die systemische Sexualtherapie rückt die Entwicklung einer erotischen Gemeinsamkeit unter Einbezug von Differenzen des sexuellen Begehrens beider Partner und der hieraus resultierenden Paardynamik in das Zentrum der Sexualtherapie. Dabei werden Techniken der systemischen Therapie z. B. zirkuläre Fragen (Fragen, welche den vermuteten Standpunkt einer Person evaluieren) oder paradoxe Interventionen (die Verordnung eines problematischen Verhaltens) etc. genutzt [33].

\section{Fazit für die Praxis}

- Auch die nicht speziell sexualmedizinisch geschulte Frauenärztin ist im klinischen Alltag mit einer Vielzahl sexueller Fragestellungen konfrontiert und kann aufgrund ihres Fachwissens sowie einer oft langfristigen Vertrauensbeziehung wertvolle Hilfestellungen zur Gestaltung einer erfüllenden Sexualität bieten.

- Im Zentrum der Unterstützung stehen eine differenzierte Information und Beratung über Auswirkungen spezifischer Erkrankungen/Therapieoptionen auf die Sexualität sowie die Durchführung einer adäquaten Sexualberatung bzw. die Vermittlung einer Sexualtherapie im Fall einer sexuellen Störung, um so auch die sexuelle Gesundheit ihrer Klientinnen zu fördern.

\section{Korrespondenzadresse}

Prof. Dr. med. B. Leeners
Clinic for Reproductive Endocrinology
Frauenklinikstrasse 10, 8091 Zurich, Schweiz
Brigitte.Leeners@usz.ch

\section{Einhaltung ethischer Richtlinien}

Interessenkonflikt. B. Leeners gibt an, dass kein Interessenkonflikt besteht.

Dieser Beitrag beinhaltet keine von den Autoren durchgeführten Studien an Menschen oder Tieren.

\section{Literatur}

\section{Verwendete Literatur}

1. Butzer B, Campbell L (2008) Adult attachment, sexual satisfaction, and relationship satisfaction: a study of married couples. Pers Relatsh 15:141-154. doi:10.1111/j.14756811.2007.00189.x
2. Byers ESE (2005) Relationship satisfaction and sexual satisfaction: a longitudinal study of individuals in long-term relationships. J Sex Res 42:113-118. doi:10.1080/ 00224490509552264

3. Weijmar Schultz W, Basson R, Binik $Y$ et al (2005) Women's sexual pain and its management. J Sex Med
2:301-316. doi:10.1111/j.17436109.2005.20347.x

4. Davison SL, Bell RJ, LaChina M et al (2009) Original Reasearch - Psychology: the relationship between self-reported sexual satisfaction and general wellbeing in women. J Sex Med 
6:2690-2697. doi:10.1111/j.17436109.2009.01406.x

5. Gallicchio L, Schilling C, Tomic D et al (2007) Correlates of sexual functioning among mid-life women. Climacteric 10:132-142. doi:10. 1080/13697130601167956

6. Hauch M (2013) Paartherapie bei sexuellen Störungen. Thieme, Stuttgart

7. Masters WH, Masters VJ (1986) Human sexual response. Bantam Books, New York

8. Hite S (2004) The hite report: a nationwide study of female sexuality. Seven Stories Press, New York

9. Kinsey AC et al (1953) Sexual behavior in the human female. Saunders, Philadelphia

10. Büsing $S$, Hoppe C, Liedtke R (2001) Sexual satisfaction of women development and results of a questionnaire. Psychother Psychosom Med Psychol 51:68-75. doi:10. 1055/s-2001-10757

11. Hurlbert DF, Apt C, Rabehl SM (1993) Key variables to understanding female sexual satisfaction: an examination of women in nondistressed marriages. J Sex Marital Ther 19:154-165. doi:10.1080/ 00926239308404899

12. Lloyd EA (2009) The case of the female orgasm. Harvard University Press, Cambridge

13. Basson R (2000) The female sexual response: a different model. J Sex Marital Ther 26:51-65. doi:10. 1080/009262300278641

14. Davis SR, Guay AT, Shifren JL, Mazer NA (2004) Endocrine aspects of female sexual dysfunction. J Sex Med 1:82-86. doi:10.1111/j.17436109.2004.10112x

15. Leeners $B$ (2013) Weibliche Libido - eine Frage der Hormone. Praxis 102:523-528. doi:10.1024/16618157/a001276

16. Davis SR, Davison SL, Donath S, Bell RJ (2005) Circulating androgen levels and self-reported sexual function in women. JAMA 294:91-96. doi:10.1001/jama.294. 1.91

17. Wierman ME (2014) Androgen therapy in women: a reappraisal:an
Endocrine Society clinical practice guideline. J Clin Endocr Metab 99(10):3489-3510. doi:10.1210/jc. 2014-2260

18. Leeners B, Kruger THC, Brody $S$ et al (2013) The quality of sexual experience in women correlates with post-orgasmic prolactin surges: results from an experimental prototype study. Sex Med 10:1313-1319. doi:10. 1111/jsm.12097

19. Angst J, Hengartner MP, Rössler W et al (2015) A Swiss longitudinal study of the prevalence of, and overlap between, sexual problems in men and women aged 20 to 50 years old. J Sex Res 52:1-11. doi:10. 1080/00224499.2014.1002556

20. Leeners B, Hengartner MP, AjdacicGross V et al (2015) Dyspareunia in the context of psychopathology, personality traits, and coping resources: results from a prospective longitudinal cohort study from age 30 to 50. Arch Sex Behav. doi:10. 1007/s10508-014-0395-y

21. The National Institute of Health and Care Excellence (NICE) Guideline. Menopause: Diagnosis and Management. https://www.nice.org. uk/guidance/ng23. Zugegriffen: 30.10.2016

22. Leeners B, Hengartner MP, Rössler $W$ et al (2014) The role of psychopathological and personality covariates in orgasmic difficulties: a prospective longitudinal evaluation in a cohort of women from age 30 to 50. J Sex Med 11:2928-2937. doi:10.1111/jsm.12709

23. Ciaplinskiene $L$, Žilaitienè $B$, Verkauskienè $R$, Žalinkevičius $R$, Bumbulienè Z, Vanagienè V, Bitzer $J$ (2016) Effect of a drospirenone-containing combined oral contraceptive on female sexual function: a prospective randomised study. Eur J Contracep Repr 21(5):395-400. doi:10.1080/ 13625187.2016.1217324

24. Mark KP, Leistner CE , Garcia JR (2016) Impact of Contraceptive Type on Sexual Desire of Women and of Men Partnered to Contraceptive Users. J Sex Med
13(9):1359-1368. doi:10.1016/j. jsxm.2016.06.011

25. Boozalis A, Tutlam, NT, Robbins CC, Peipert JF (2016) Desire and Hormonal Contraception. Obstet Gynecol 127(3):563-572. doi:10. 1097/AOG.0000000000001286

26. von Sydow K (1999) Sexuality during pregnancy and after childbirth: a metacontent analysis of 59 studies. J Psychosom Res 47:27-49

27. von Sydow K, Ullmeyer M, Happ N (2001) Sexual activity during pregnancy and after childbirth: results from the sexual preferences questionnaire. JPsychosom Obstet Gynaecol 22:29-40. doi:10.3109/ 01674820109049948

28. Kavanagh J, Kelly AJ, Thomas $J$ (2001) Sexual intercourse for cervical ripening and induction of labour. Cochrane Database Syst Rev. doi:10.1002/14651858. CD003093

29. Buhling KJ, Schmidt S, Robinson JN et al (2006) Rate of dyspareunia after delivery in primiparae according to mode of delivery. Eur J Obstet Gynecol Reprod Biol 124:42-46. doi:10.1016/j.ejogrb.2005.04.008

30. Schytt E, Lindmark G, Waldenström U (2005) Physical symptoms after childbirth: prevalence and associations with self-rated health. BJOG 112:210-217. doi:10.1111/j.14710528.2004.00319.x

31. Bitzer J, Platano G, Tschudin S, Alder J (2007) Sexual counseling for women in the context of physical diseases: a teaching model for physicians. J Sex Med 4:29-37. doi:10.1111/j.1743-6109.2006. 00395.x

32. Buddeberg C (2005) Sexualberatung. Thieme, Stuttgart

33. Clement U (2016) Systemische Sexualtherapie. Klett-Cotta, Stuttgart

\section{Weiterführende Literatur}

34. Young LJ, Wang Z (2004) The neurobiology of pair bonding. Nat Neurosci 7:1048-1054. doi:10. 1038/nn1327 
Teilnahme am zertifizierten Kurs auf CME.SpringerMedizin.de

- Der Teilnahmezeitraum beträgt 12 Monate, den Teilnahmeschluss finden Sie online beim CME-Kurs.

- Fragen und Antworten werden in zufälliger Reihenfolge zusammengestellt.

- Pro Frage ist jeweils nur eine Antwort zutreffend.

- Für eine erfolgreiche Teilnahme müssen 70\% der Fragen richtig beantwortet werden.

Welche Aussage zur Erregung bei der Frau trifft zu? Bei Erregung ...

O entspricht die subjektive erlebte Erregung den physiologisch messbaren Veränderungen.

$\bigcirc$ ist ein Anspannen der Beckenbodenmuskulatur zu jedem Zeitpunkt pathologisch.

- kommt es ausschließlich im Genitalbereich zu einer Hyperämie.

○ führt eine Hyperämie per Transsudation zur Lubrikation der Vagina.

$\bigcirc$ entsteht bei Frauen zunehmend der Wunsch nach Geschlechtsverkehr.

?ie lange dauert der weibliche Orgasmus durchschnittlich?

O $5 \mathrm{~s}$

O $15 \mathrm{~s}$

○ $30 \mathrm{~s}$

○ $60 \mathrm{~s}$

○ $90 \mathrm{~s}$

? Welches Hormon ist hauptsächlich an der Regulation der physiologischen Voraussetzungen für eine erfüllende Sexualität im Bereich der Vaginalwand beteiligt?

○ Testosteron

O Prolaktin

O Progesteron

Östrogen

TSH (Thyreoidea-stimulierendes Hormon)
Welche ist die häufigste sexuelle Störung bei der Frau?

Orgasmusstörung

○ Libidostörung

○ Vaginismus

O Dyspareunie

$\bigcirc$ Erregungsstörung

?elche Aussage über den Vaginismus trifft zu?

Eine Introitusplastik führt zu einer Verbesserung der Symptomatik.

O Es handelt sich um eine unwillkürliche Anspannung des Beckenbodens, die therapieresistent ist.

Os liegt eine Fibrosierung des Introitus vor.

Es ist nur der Versuch eines Geschlechtsverkehrs, nie jedoch eine gynäkologische Untersuchung von dieser Symptomatik betroffen.

○ Die Störung kann über das Erlernen einer bewussten Kontrolle des Beckenbodentonus aufgelöst/reduziert werden.

Welche der folgenden sexuellen Aktivitäten sollte im komplikationslosen dritten Trimenon vermieden werden - auch wenn von beiden Partnern gewünscht?

○ Petting

O Geschlechtsverkehr in Seitenlage

O Geschlechtsverkehr in Missionarsstellung

○ Brustzärtlichkeiten

Intensive Küsse
? Welche Aussage zu einer Karzinomerkrankung trifft zu?

○ Ausschließlich körperliche Veränderungen und Nebenwirkungen der Therapie führen zu ungünstigen Wirkungen auf die Sexualität.

Es kommt grundsätzlich zu einer langfristigen Verschlechterung der Sexualität.

O Sexualität ist auch in der Akutphase ein zentrales Thema für die Betroffenen.

Es treten psychische, soziale und somatische Auswirkungen auf.

○ Frauen sollten während einer Radiatio auf gar keinen Fall Geschlechtsverkehr haben.

? Welche Aussage zur Sexualberatung ist richtig?

O Sie kann ausschließlich von speziell geschulten Sexualmedizinern durchgeführt werden.

O Sie sollte erst auf ausdrückliches Nachfragen der Patientin durchgeführt werden.

O Sie sollte spezifisch sexuelle Begriffe indirekt umschreiben und andeuten.

O Sie darf auf keinen Fall eine Exploration von spezifischen Details der partnerschaftlichen Sexualität beinhalten.

○ Sie kann auf der Basis des gynäkologischen Fachwissens bereits wichtige Hilfestellungen in einer sexuell schwierigen Situation bieten. 
Sollte eine Patientin ein Gleitmittel benötigen, welches der folgenden bietet dann den höchsten Anwendungskomfort?

O Vaseline

○ Wasserlösliches Gleitmittel

○ Fetthaltiges Gleitmittel

O Gleitmittel auf Silikonbasis

O Speichel
Eine Sexualtherapie ...

kann von jeder/m Arzt/Ärztin unabhängig von der Fachrichtung problemlos angeboten werden.

$\bigcirc$ dauert bei adäquatem Vorgehen mindestens ein Jahr.

O sollte bei sexuellen Störungen, die sich nach einer Sexualberatung nicht gebessert haben, angeboten werden.

○ führt auf jeden Fall zu einer Verbesserung der Symptomatik, wenn sie entsprechend der Richtlinien einer etablierten sexualmedizinischen Schule umgesetzt wird.

O sollte idealerweise mit beiden Partnern getrennt durchgeführt werden.

\section{$\underline{\underline{0}}$}

\section{CME-Punkte sammeln in 3 Schritten}

Als Zeitschriftenabonnent stehen Ihnen unter CME.SpringerMedizin.de alle zertifizierten Fortbildungskurse Ihrer Zeitschrift zur Verfügung.

\section{So einfach sammeln Sie CME-Punkte:}

\section{> Registrieren}

Um CME-Fortbildungen bearbeiten zu können, müssen Sie sich einmalig unter www.springermedizin.de/register registrieren. Bitte geben Sie bei der Registrierung die Lieferadresse Ihrer abonnierten Zeitschrift an, damit die Angaben Ihres OnlineAccounts mit den Angaben Ihres Zeitschriften-Abonnements übereinstimmen. Die CME-Beiträge werden anschließend automatisch freigeschaltet.

Beitrag auswählen

Sobald Sie sich mit Ihren Zugangsdaten angemeldet haben können Sie auf CME.SpringerMedizin.de die gewünschten CME-Kurse Ihrer Zeitschrift nutzen. Die Kurse können jederzeit unterbrochen und später fortgesetzt werden.
CME-Punkte sammeln

Zu jedem Fortbildungskurs gehört ein Fragebogen mit 10 CMEFragen. Mit 7 richtigen Antworten haben Sie bestanden und erhalten umgehend eine Teilnahmebescheinigung!

Teilnehmen und weitere Informationen unter:

CME.SpringerMedizin.de

Unser Tipp: Noch mehr Fortbildung bieten die e.Med-KombiAbos. Hier stehen Ihnen die CME-Kurse der Fachzeitschriften von Springer Medizin in elektronischer Form zur Verfügung. Auf Wunsch erhalten sie mit den e.Med-Kombi-Abos außerdem eine gedruckte Fachzeitschrift Ihrer Wahl.

Testen Sie e.Med 30 Tage kostenlos und unverbindlich!

Jetzt informieren unter

www.springermedizin.de/eMed oder telefonisch unter 0800-77 80777

(Montag bis Freitag, 10 bis 17 Uhr) 
Hier steht eine Anzeige.

黛 Springer 